\title{
Actinomyces lymphadenitis
}

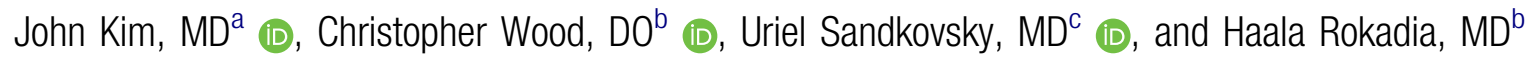 \\ ${ }^{a}$ Department of Internal Medicine, Baylor University Medical Center, Dallas, Texas; ${ }^{\circ}$ Division of Pulmonary and Critical Care Medicine, \\ Baylor University Medical Center, Dallas, Texas; 'Division of Infectious Diseases, Baylor University Medical Center, Dallas, Texas
}

\begin{abstract}
Actinomyces is a gram-positive anaerobe that colonizes the human oral cavity. Its pathogenesis involves the disruption of the mucosal membrane, leading to suppuration and subsequent abscess formation, most commonly in the cervicofacial region. The bacteria form masses consisting of aggregates of branching, filamentous bacilli. They typically spread by direct tissue invasion and less commonly through hematogenous spread. Lymphatic spread is extremely uncommon. To the best of our knowledge, only two cases have been reported with lymph node involvement. Clinically, lymphadenopathy associated with Actinomyces may be misinterpreted as malignancy, causing unnecessary surgical interventions when only antibiotics are warranted. This case highlights the importance of properly diagnosing a rare phenomenon of Actinomyces lymphadenitis.
\end{abstract}

KEYWORDS Actinomyces; lymph node; pulmonary actinomycosis

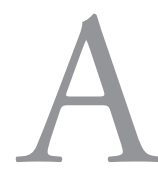

ctinomyces is a filamentous gram-positive bacillus that spreads by direct tissue invasion. Lymphatic spread is uncommon. To the best of our knowledge, only two cases have been reported with lymph node involvement. ${ }^{1,2}$ We herein report a rare case of Actinomyces lymphadenitis.

\section{CASE REPORT}

A 37-year-old man with a history of smoking, obstructive sleep apnea, and gastroesophageal reflux presented with a 3-day history of fever, cough, and small-volume hemoptysis despite recent treatment with azithromycin. He was hemodynamically stable and denied chills, night sweats, dyspnea, rashes, sick contacts, and recent anticoagulation use. He had poor dentition but no oropharyngeal bleeding. The lungs were clear.

Noncontrast computed tomography (CT) showed bibasilar ground-glass opacities. The patient was empirically treated with vancomycin and piperacillin/tazobactam. Bronchoscopy with bronchoalveolar lavage was performed. No endobronchial lesions were identified, but old blood was seen in the right lower lobe subsegment. An initial Gram stain of respiratory cultures revealed occasional gram-positive cocci and rods. However, respiratory cultures were negative for any bacterial growth. Repeat CT of the chest with contrast showed air in the subcarinal lymph node concerning for mediastinitis vs esophageal perforation (Figure 1a, Figure 1b). The patient underwent esophagogastroduodenoscopy, where no esophageal lesions were seen but an irregularly hypoechoic lymph node was identified and aspirated via esophageal ultrasonography. The initial Gram stain of the lymph node revealed grampositive cocci in pairs, but final cultures were positive for Actinomyces. The patient improved clinically with antibiotics and was discharged home with piperacillin/tazobactam. Repeat CT during the 1-month follow-up showed residual disease with moderate improvement (Figure 1c). The patient was continued on piperacillin/tazobactam for 8 weeks, followed by 6 months of doxycycline.

\section{DISCUSSION}

Actinomycosis is a chronic disease caused by Actinomyces, an anaerobic gram-positive bacteria that normally colonizes in the human mouth, digestive tract, and urogenital tract. ${ }^{1-5}$ Typical clinical presentations include cervicofacial actinomycosis following dental procedures, pelvic actinomycosis in women with an intrauterine device, and pulmonary actinomycosis in smokers with poor dentition. ${ }^{5}$ Actinomyces found in lymph nodes is uncommon, with only two reported cases. $^{1,2}$ Due to the rarity, lymphadenopathy with Actinomyces is commonly misdiagnosed as malignancy, causing unnecessary surgical interventions when only antibiotics are warranted. ${ }^{2}$ The finding of Actinomyces in the subcarinal

Corresponding author: John Kim, MD, Department of Internal Medicine, Baylor University Medical Center, 3500 Gaston Ave., Dallas, TX 75246 (e-mail: john.kim1@bswhealth.org)

Received January 1, 2020; Revised March 10, 2020; Accepted March 16, 2020. 


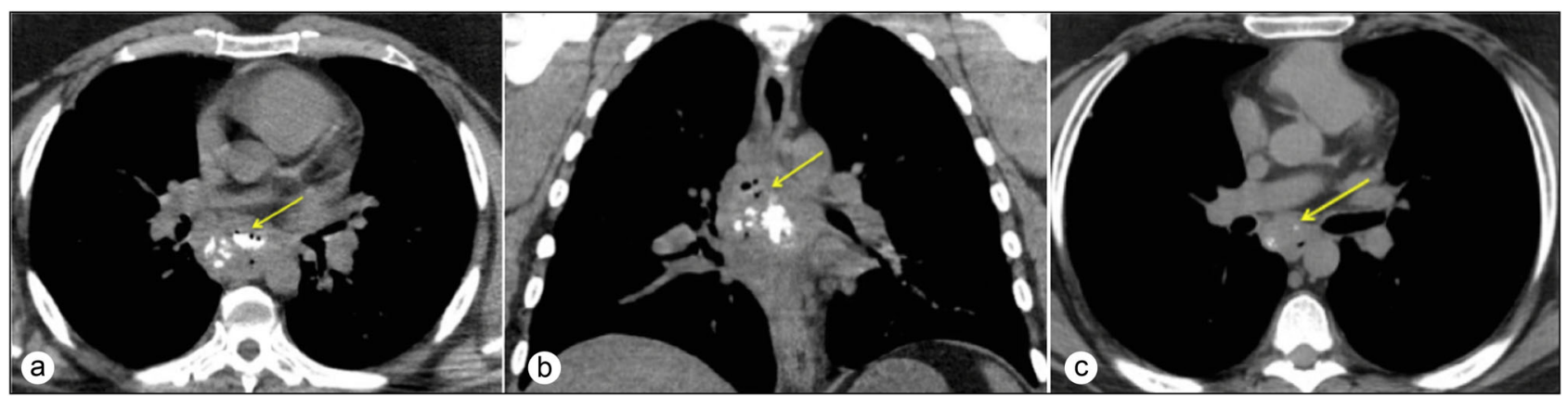

Figure 1. CT of the chest. (a) Axial and (b) coronal views showing air in the subcarinal lymph node. (c) A scan after antibiotic treatment showing residual disease and improvement.

lymph node with concomitant cough and hemoptysis makes our case very rare.

The pathogenesis of Actinomyces is through the disruption of mucosal membranes, leading to suppuration and subsequent abscess formation. ${ }^{1-4}$ The bacteria spread by directly invading local tissues. In our case, the initial respiratory Gram stain was positive for occasional gram-positive rods. Given our patient's smoking history, poor dentition, and ground-glass opacities on CT, he likely initially developed early stages of pulmonary actinomycosis, which then spread through the lymphatic system, infecting the subcarinal lymph nodes.

The diagnosis of actinomycosis is made by bacterial culture and pathology. Prolonged bacterial cultures in anaerobic conditions are necessary for identification of the bacterium. ${ }^{3}$ Typical microscopic findings include necrosis with yellowish sulfur granules and a filamentous gram-positive fungal-like pathogen. ${ }^{3}$ Specifically, in lymph nodes, histologic features of Actinomyces include fibrous thickening of the capsule, nodulation with fibrous bands, and multiple abscesses with central loculations and sulfur granules. ${ }^{2}$ Patients with actinomycosis require prolonged high doses of penicillin $G$ or amoxicillin. ${ }^{3,6,7}$ Alternatives are ceftriaxone, doxycycline, macrolides, and clindamycin. ${ }^{4-7}$ Our patient was given piperacillin/tazobactam due to convenience of continuous infusion as well as to cover other anaerobic bacteria.

\section{ORCID}

John Kim (D) http://orcid.org/0000-0002-4672-5440

Christopher Wood (iD http://orcid.org/0000-0003-4503-2076

Uriel Sandkovsky iD http://orcid.org/0000-0001-9077-3426

Haala Rokadia (iD http://orcid.org/0000-0002-3740-2359

1. Amrikachi M, Krishnan B, Finch CJ, Shahab I. Actinomyces and Actinobacillus actinomycetemcomitans-Actinomyces-associated lymphadenopathy mimicking lymphoma. Arch Pathol Lab Med. 2000;124: 1502-1505.

2. Arik D. Actinomyces lymphadenitis: case report. Turk Patoloji Derg. 2013;29:80-82

3. Ferry T, Valour F, Karsenty J, et al. Actinomycosis: etiology, clinical features, diagnosis, treatment, and management. Infect Drug Resist. 2014;7:183-197. doi:10.2147/IDR.S39601.

4. Könönen E, Wade W. Actinomyces and related organisms in human infections. Clin Microbiol Rev. 2015;28:419-442. doi:10.1128/CMR. 00100-14

5. Reichenbach J, Lopatin U, Mahlaoui N, et al. Actinomyces in chronic granulomatous disease: an emerging and unanticipated pathogen. Clin Infect Dis. 2009;49:1703-1710.

6. Branquinho D, Andrade D, Almeida N, Sofia C. Mediastinitis by Actinomyces meyeri after esophageal stent placement. BMJ Case Rep. 2014;2014:1-3. doi:10.1136/bcr-2014-204499.

7. Walther K, Bruder E, Goldenberger D, Mayr J, Schaad U, Ritz N. Actinomyces neuii isolated from a 20-month-old girl with cervical lymphadenitis. J Pediatric Infect Dis Soc. 2015;4:32-37. 